\title{
EXTENDED X-RAY BREMSSTRAHLUNG ISOCHROMAT FINE STRUCTURE
}

\author{
E. SOBCZAK \\ Institute of Physics, Polish Academy of Sciences \\ Al. Lotników 32/46, 02-668 Warszawa, Poland
}

(Received October 12, 1992)

\begin{abstract}
Main aspects concerning a new method of extended X-ray bremsstrahlung isochromat fine structure (EXBIFS) are specified and discussed. The EXBIFS effect is studied here by application of a single-scattering theory, which explains well the experimental phenomenon that EXBIFS of $\mathrm{Cu}$ and $\mathrm{Pd}$ is strikingly similar to the $p$ partial density of states, although the $s$ - and $d$-symmetry contributions are not negligible. The single-scattering model of EXBIFS has been successfully applied for explanation of temperature effects resulting in a smearing of oscillations for big $k$ values. It is established here that interatomic distances can be evaluated from EXBIFS by means of the single-scattering theory and the Fourier analysis.
\end{abstract}

PACS numbers: $79.20 . \mathrm{Kz}, 78.70 . \mathrm{Dm}$

\section{Introduction}

X-ray radiation was discovered in 1895 by Röntgen [1] and was soon applied in many fields. During the next twenty five years, the main X-ray characteristics and phenomena were identified and investigated, e.g., polarization of X-rays, frequency of X-rays, difference between characteristic and bremsstrahlung X-rays, $\mathrm{X}$-ray diffraction by crystals, and the Compton effect.

The quantum nature of light, being one of the fundamental phenomena of physics, provided the main reason for initiation of X-ray bremsstrahlung isochromat spectroscopy (BIS). Planck's constant had been qualitatively estimated by Einstein in 1905 from observations of the ultraviolet photoemission effect [2]. Precise evaluation of Planck's constant became possible in 1915 by means of X-ray BIS studies, first done by Duane and Hunt [3]. The BIS-based method for determination of $h / e$ was improved by several authors and remained the most accurate method for evaluation of Planck's constant until Josephson's experiment in 1969.

Through more than twenty years following Duane and Hunt's work, physicists approximated the threshold of an isochromat by a straight line extrapolation. This was acceptable because the measurements of the isochromats were 
TABLE I

Milestones in the origination and development of bremsstrahlung isochromat spectroscopy.

\begin{tabular}{|c|c|c|}
\hline Year & Subject & Reference \\
\hline 1895 & discovery of X-rays & Röntgen [1] \\
\hline 1905 & evidence of quantum nature of light & Einstein [2] \\
\hline 1907 & estimation of X-ray frequency & Wien [8] \\
\hline 1912 & diffraction of X-rays & $\begin{array}{l}\text { Laue et al. [9], } \\
\text { Bragg [10] }\end{array}$ \\
\hline 1915 & $\begin{array}{l}\text { evaluation of the Planck } \\
\text { constant using X-ray BIS }\end{array}$ & Duane and Hunt [3] \\
\hline 1918 & $\begin{array}{l}\text { spectral distribution of } \\
\text { bremsstrahlung }\end{array}$ & $\begin{array}{l}\text { Urley [11], } \\
\text { Kuhlenkampff [12] }\end{array}$ \\
\hline 1923 & classical theory of bremsstrahlung & Kramers [13] \\
\hline 1931 & $\begin{array}{l}\text { nonrelativistic quantum theory } \\
\text { of bremsstrahlung }\end{array}$ & Sommerfeld [14] \\
\hline 1941 & $\begin{array}{l}\text { discovery of the pronounced BIS } \\
\text { structure (Ohlin's maximum) }\end{array}$ & $\begin{array}{l}\text { Ohlin [4], } \\
\text { Bearden and Schwarz [5] }\end{array}$ \\
\hline 1945 & $\begin{array}{l}\text { explanation of BIS maximum via band } \\
\text { structure of solids }\end{array}$ & Lindhard [6], Nijboer [7] \\
\hline 1953 & characteristic X-ray BIS & Nilsson [15] \\
\hline 1954 & soft X-ray BIS & Gerthsen and Albert [16] \\
\hline 1960 & $\begin{array}{l}\text { theory of energy-dependent } \\
\text { probability of BIS transitions }\end{array}$ & Kessler and Ulmer [17] \\
\hline 1962 & $\begin{array}{l}\text { shift of the BIS threshold due to } \\
\text { energy gap and band bending }\end{array}$ & Auleytner and Lidén [18] \\
\hline 1965 & $\begin{array}{l}\text { development of soft X-ray } \\
\text { spectrometry for BIS }\end{array}$ & Eggs and Ulmer [19] \\
\hline 1969 & $\begin{array}{l}\text { photon energy dependence of the } \\
\text { bremsstrahlung isochromat shape }\end{array}$ & Böhm and Ulmer [20] \\
\hline 1970 & $\begin{array}{l}\text { determination of the work function } \\
\text { by BIS }\end{array}$ & Merz $[21]$ \\
\hline 1973 & localization of the Fermi level & Mechelke and Ulmer [22] \\
\hline 1974 & $4 f$ resonance effects in BIS & Liefeld et al. [23] \\
\hline 1977 & ultraviolet BIS at $9.6 \mathrm{eV}$ & Dose [24] \\
\hline 1977 & $\begin{array}{l}\text { cross-sections for radiative } \\
\text { capture of electrons by ions }\end{array}$ & Hahn and Rule [25] \\
\hline 1979 & $\begin{array}{l}\text { BIS and XPS studies of RE } 4 f \text { levels } \\
\text { using a modified XPS apparatus }\end{array}$ & Lang et al. [26] \\
\hline
\end{tabular}


TABLE I (cont.)

\begin{tabular}{|c|c|c|}
\hline Year & Subject & Reference \\
\hline 1979 & $\begin{array}{l}\text { calculation of Ni BIS using } \\
\text { partial densities of states and } \\
\text { transition probabilities }\end{array}$ & Chu and Best [27] \\
\hline 1980 & $3 d$ resonance in BIS & Riehle [28] \\
\hline 1981 & extended structure of X-ray BIS & Sobczak et al. [29] \\
\hline 1981 & $\begin{array}{l}\text { Green's function theory of UV BIS } \\
\text { and initiation of the name "inverse } \\
\text { photoemission" }\end{array}$ & Pendry $[30]$ \\
\hline 1982 & spin-polarized inverse photoemission & Unguris et al. [31] \\
\hline 1984 & extended structure of soft X-ray BIS & Speier et al. [32] \\
\hline 1988 & $\begin{array}{l}\text { evidence of the local character of } \\
\text { BIS transitions and breakdown of } \\
\text { the total DOS image for BIS }\end{array}$ & Sobczak et al. [33] \\
\hline 1988 & matrix elements for soft X-ray BIS & Speier et al. [34] \\
\hline 1988 & matrix elements calculated for EXBIFS & Šmůnek et al. [35] \\
\hline 1989 & multiple-scattering theory of EXBIFS & Šimůnek et al. [36] \\
\hline 1989 & $\begin{array}{l}\text { UV BIS by scanning tunnelling } \\
\text { microscopy }\end{array}$ & Reihl et al. [37] \\
\hline
\end{tabular}

done at points separated by large steps. As soon as experiments had been done with smaller steps, an unexpected maximum in the neighborhood of the limit, the so-called Ohlin's maximum, was observed for several metals [4, 5]. A few years later, in 1945-46, the first rough explanation of Ohlin's maximum based on the band structure of solids was given by Lindhard [6] and Nijboer [7]. Thus, X-ray BIS studies of the electronic structure of solids were initiated in the early 1940's.

It is now known that interpretations of BIS spectra made before the late 1970's were often incorrect, because physicists wrongly trusted the total-density-of-states approximation to BIS theory. Further, they also badly overestimated the contributions of discrete characteristic electron energy losses to the fine structure of BIS. During the last 15 years, much progress has been made both in experiment and in theory relative to BIS.

Every year, more and more scientists become interested in using BIS. In fact, interest in BIS has now increased to the point that the well-known firm "Vacuum Generator" has started offering a commercial BIS spectrometer for soft X-rays as an optional accessory to the ESCA photoelectron machine.

A list of the crucial papers in the historical development of the bremsstrahlung 
isochromat spectroscopy is presented in Table I. The papers were selected on the basis of the novelty of the subjects published.

At the Institute of Physics of the Polish Academy of Sciences in Warsaw, Professor J. Auleytner and his co-workers have been developing BIS since the 1970's. Major efforts in both theory and experiment have been made in recent years, all to the end of understanding of the nature of the BIS structure. The experimental efforts have involved new BIS experiments in an extended energy range for several metals with well-known electronic structure.

Extended X-ray bremsstrahlung isochromat fine structure (EXBIFS) was first reported by Sobczak and co-workers at the International Conference on X-ray Processes and Inner-Shell Ionization held at Stirling, England in 1980. They discovered that the EXBIFS of Ge was similar to the $K$-edge absorption spectra in the extended energy range (EXAFS). At the Third International EXAFS Conference in Stanford in 1984, Speier and co-workers presented EXBIFS results for $\mathrm{Cu}$ in the soft X-ray region [32]. Soon thereafter, further new results of EXBIFS measurements were reported by authors from both groups. Photon energies equal to $5415 \mathrm{eV}$ and to $1487 \mathrm{eV}$ used to be applied by the Sobczak and by the Spcier group, respectively. It was shown that the photon energy chosen for isochromat studies had a strong influence on the fine structure of EXBIFS.

To the present time, all of the publicly reported experimental EXBIFS studies in the world have been carried out by these two groups, only. On the theory side, a considerable contribution to EXBIFS has been made by Simünek and co-workers $[35,36]$.

The author's aim in this present work is to specify and discuss in detail main aspects concerning the EXBIFS method. There are two main reasons for doing it so now. First, no book or review article covering this new subject exists now. Second, many old books and articles which do cover BIS (not EXBIFS) suffer from errors in interpretation of the X-ray bremsstrahlung isochromats.

This work presents results of EXBIFS studies for several fcc metals: $\mathrm{Cu}, \mathrm{Ni}$, Co, Pd, and Ag. Experimental and theoretical comparisons between EXBIFS and EXAFS are presented. It is shown that interatomic distances can be determined from EXBIFS with the aid of the Fourier analysis. The EXBIFS effect is theoretically explained by application of a single-scattering theory of EXBIFS and the theoretical transition probabilities for BIS. The single-scattering theory is shown to be very successful in estimation of the temperature smearing of EXBIFS.

\section{Bremsstrahlung radiation and photoeffect as inverse processes}

Bremsstrahlung experiments are divided into two groups regarding the photon energy involved: ultraviolet and X-ray radiation ranges. It is an analogy to the UV and X-ray photoemission.

One of the reasons for such classification is the momentum resolution due to the angular divergence of the electron beam. A typical angular divergence $2^{\circ}$ of the electron beam is small enough to get a quite good momentum resolution in UV bremsstrahlung experiments and much too big to resolve the X-ray bremsstrahlung transitions in the momentum space. 
Another reason is the momentum conservation rule. The UV photon momentum is negligibly small relative to the dimension of the Brillouin zone, therefore a direct transition approximation is valid for UV BIS. This approximation is not valid for the X-ray BIS, because the X-ray photon momentum is comparable with the size of the Brillouin zone. Therefore UV BIS gives the opportunity to study electronic bands as a function of momentum, opposite to the X-ray BIS which integrates the electronic structure all over the Brillouin zone.

A smaller mean free path for inelastic scattering of primary electrons in the UV BIS makes this method more sensitive for surface studies than the X-ray BIS spectroscopy. Therefore, non-occupied both surface and bulk electron states can be studied by UV BIS.

Quite important reason for the UV and X-ray BIS differentiation is the localization of the X-ray bremsstrahlung transition to the small space around the nucleus due to a big momentum transfer. In contrary to the X-ray BIS, the UV bremsstrahlung transitions occur all over the space and can be treated in a similar way as the spontaneous photon emission in the optical range.

The bremsstrahlung process is treated at some conditions as a time-reversed phatoelectron effect (Fig. 1) and therefore named inverse photoemission [30]. The

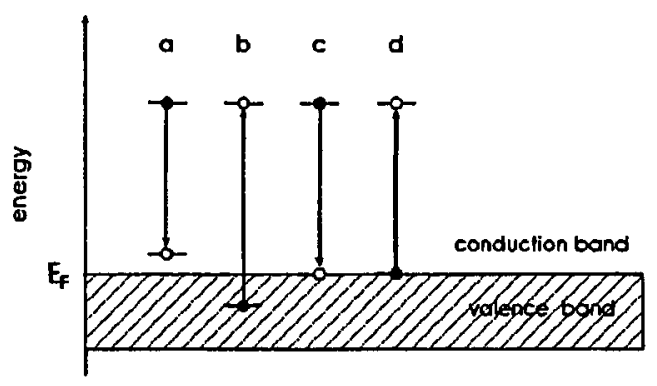

Fig. 1. The bremsstrahlung process ( $a$ and $c$ ) can be treated as time-reversed to the photoelectron process from valence band ( $b$ and $d$ ), therefore the same computing formalism based on the band structure theory can be used for both phenomena.

inversion relation is nearly fulfilled in UV photon range except the condition that in the bremsstrahlung final state one electron is added to an atom, while in the photoeffect final state one electron is lacking in an atom. A similar inversion relation also occurs in the X-ray photon range between BIS and photoemission from valence band. Therefore, the bremsstrahlung spectra can be calculated theoretically as time-reversed photoemission.

There is no simple inversion relation between $\mathrm{X}$-ray bremsstrahlung radiation and X-ray absorption near absorption edges, however the both phenomena correspond to each other in a large extent, which is shown in this work. Both X-ray BIS and X-ray absorption spectra give information on the unoccupied electron states in any energy range above the Fermi level. X-ray BIS and X-ray absorption transitions can involve final states of the same energy, but not of the same symmetry. The initial electron state is quite different in BIS and X-ray absorption. It is a 
nearly-free-electron state in BIS and a core state with a well-defined orbital quantum number $l$ in the X-ray absorption. Therefore, the selection rules for electron transitions are simple and known for $\mathrm{X}$-ray absorption, but rather complicated for BIS due to a strong dependence on the atomic number and photon energy.

The physical property of X-ray spectra is a broadening due to the lifetime of a core hole. For example, the $K$ Pd hole lifetime makes a broadening of $6.8 \mathrm{eV}$ of the absorption spectrum, which does not exist in BIS.

The BIS intensity is a sum over all types of atoms, while an X-ray absorption spectrum results from only one element (it can happen, specially for low-energy absorption edges, that X-ray absorption spectra from different elements overlap in the photon energy scale).

\section{Experimental details}

The X-ray bremsstrahlung emission from an X-ray tube target results from retardation of electrons bombarding the target from the cathode. For the X-ray tube voltage $U$ the initial state of these electron transitions is at the energy level

$$
E_{\mathrm{i}}=e U+\phi,
$$

where $\phi$ is the work function of the cathode, $e$ is the electron charge, and the zero of energy is taken at the Fermi level $E_{\mathrm{F}}$ of the target. The final electron state is an empty state at the energy $E_{\mathrm{f}}$ above the Fermi level (see Fig. 2). The electron

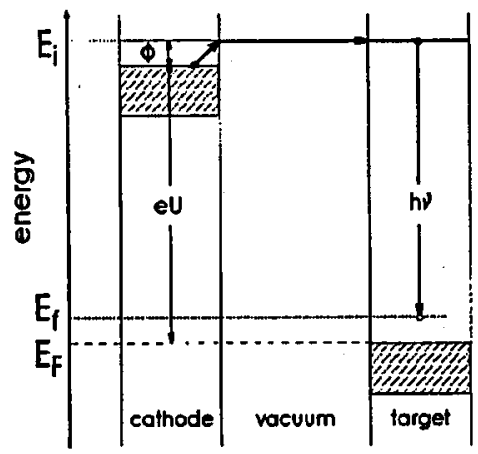

Fig. 2. Scheme of energy levels of electrons in the X-ray tube at the bremsstrahlung transition.

transition between the initial and final states produces a photon of the energy $h \nu$ :

$$
h \nu=E_{\mathrm{i}}-E_{\mathrm{f}},
$$

where $\nu$ is the photon frequency and $h$ is Planck's constant. Bremsstrahlung transitions to the lowest available final states give the short-wave limit $\nu_{0}$ of the bremsstrahlung spectrum.

The bremsstrahlung spectra can be measured either in an isochromat mode (BIS) or constant initial state mode. Usually, the isochromat method is applied. In this method an X-ray monochromator is set to pass a narrow band of radiation 
at a fixed frequency $\nu$. The intensity of the bremsstrahlung radiation is recorded as a function of voltage $U$. The voltage $U$ is increased step by step, therefore the energy of the final electron state also increases step by step following the voltage.

An X-ray bremsstrahlung isochromat spectrometer used in this work was constructed by Professor Auleytner's group in the Institute of Physics of the Polish Academy of Sciences in Warsaw. The instrument consists of a dismountable X-ray tube, a high-stabilized high-voltage supply, and an X-ray Johann-type monochromator.

The sample is mounted on a water-cooled target of an Ohlin-type X-ray tube [15]. The X-ray tube is not placed on the Rowland circle, but shifted towards the crystal. The target-crystal distance is $120 \mathrm{~mm}$ only. The electron emission current from the directly heated thoriated tungsten cathode is usually in the range 10-15 mA. Zeolite sorption pumps and an ion pump keep the vacuum in the $\mathrm{X}$-ray tube at $10^{-4} \mathrm{~Pa}$. The vacuum in the spectrometer chamber is $10^{-1} \mathrm{~Pa}$. The advantage of using a proportional counter as a detector is a very low background equal to 16 counts/min.

The high voltage is stabilized with accuracy $2 \times 10^{-5}$. Three stages of the high-voltage changing is available. The first setting done by hand before turning on makes it possible to choose the voltage in the range from about $2 \mathrm{kV}$ to about $9 \mathrm{kV}$ with discrete values $125 \mathrm{~V}$. The second setting is to tune the voltage by hand in a continuous mode in the smaller range of $600 \mathrm{~V}$ when the supply is turned on. The third one is to increase the voltage automatically step by step in the range up to $140 \mathrm{~V}$. The combination of all modes makes it possible to measure BIS in the extended range of voltage.

In the first EXBIFS experiment [29] performed for germanium, the monochromator used was a bent quartz (1010) crystal. The total spectral resolution of the quartz spectrometer was estimated to be about $3.5 \mathrm{eV}$.

Later, the spectrometer resolution has been much improved by applying the $\operatorname{Si}(220)$ crystal with the interplanar distance $d_{220}=1.92015 \AA$. The $\mathrm{Si}(220)$ crystal is bent elastically in a steel holder to a cylindrical curvature of $1 \mathrm{~m}$ radius. The spectrometer calibration is done using the $\mathrm{Cr} K_{\alpha_{1}}$ emission line (the photon energy equal to $5414.72 \mathrm{eV}$ ) from a $\mathrm{Cr}$ plate excited by $7.4 \mathrm{keV}$ electrons. The $\mathrm{Cr} K_{\alpha_{1,2}}$ dublet is fully resolved and the measured FWHM of the Cr $K_{\alpha_{1}}$ is $3.3 \mathrm{eV}$, while the natural width of this line is $2.07 \mathrm{eV}$ [38].

The width of the total spectrometer function FWHM $=1.8 \mathrm{eV}$, including the monochromator window and the energy spread of electrons emitted from the cathode, is estimated from the broadening of the Pd BIS threshold measured up to $20 \mathrm{eV}$ above $E_{\mathrm{F}}$ in $0.2 \mathrm{eV}$ steps. The Pd BIS threshold is shown in Fig. 3 together with the $\operatorname{Pd} L_{1}$ X-ray absorption near-edge structure (XANES) obtained by Davoli et al. [39] using synchrotron radiation. The advantage of the BIS spectrum is that the BIS spectrum is not broadened by the natural width of a core level, which could be quite big e.g. about $6 \mathrm{eV}$ in the case of the $\mathrm{Pd} L_{1}$ level.

The BIS spectra from the fcc metals were measured with $0.5 \mathrm{~V}$ or $1 \mathrm{~V}$ steps up to about $300 \mathrm{~V}$ above the threshold. The targets were $0.2-0.25 \mathrm{~mm}$ thick foils. The incident electron beam was constant all over the energy range. Several scans of isochromats were summarized. The power of the $\mathrm{X}$-ray tube was experimentally 


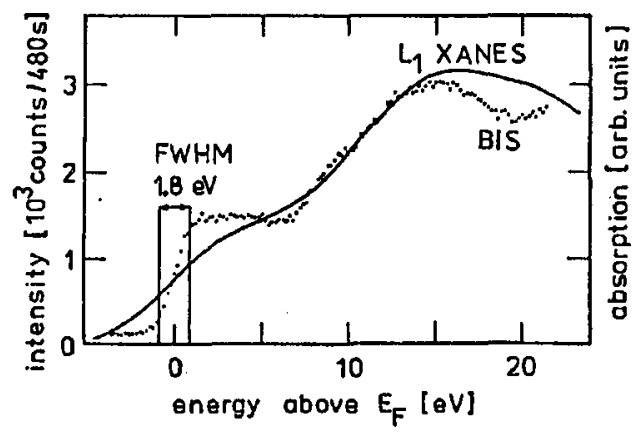

Fig. 3. The total resolution of the Pd BIS spectrum (dots) is higher than that of the $L_{1}$ XANES spectrum of Pd (solid line).

chosen not to melt the sample, but to keep enough high temperature of the surface in order to minimize surface contaminations during long-time measurements. Small changes in the shape as well as the lowering of the intensity of $\mathrm{Ni}, \mathrm{Co}$, and $\mathrm{Ag}$ isochromats were observed during the 40 hours measurement, due to the carbon contamination.

The results presented in Fig. 4 show that the BIS spectra of metals studied have really an extended structure. A smooth arbitrary curve displaying the basal shape of an isochromat was found by computer fitting. Intensity oscillations

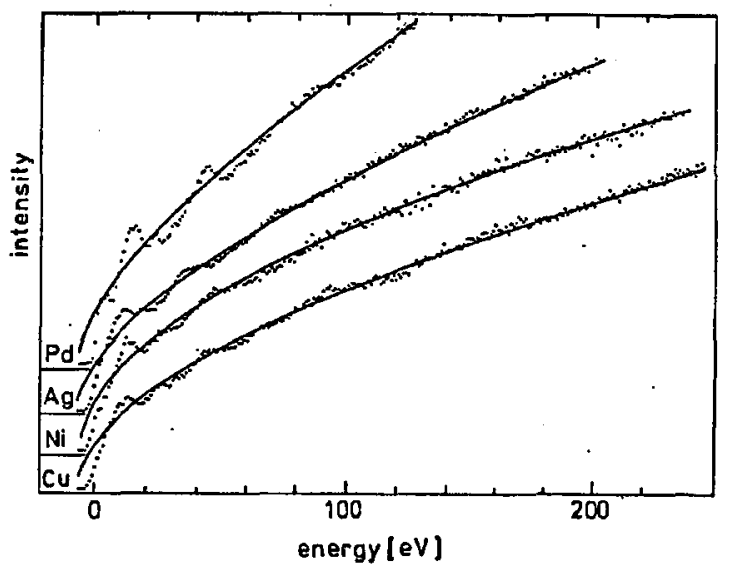

Fig. 4. The X-ray bremsstrahlung isochromats of $\mathrm{Pd}, \mathrm{Ag}, \mathrm{Ni}$, and $\mathrm{Cu}$ at the photon energy of $5415 \mathrm{eV}$ (points) and arbitrary smooth curves (solid lines) displaying the basal shape of isochromat (by Sobczak et al. [40]).

(EXBIFS) were obtained as a result of subtraction of a smooth curve from each isochromat. In Fig. 5 the Pd EXBIFS oscillations are compared with the theoretical electronic band structure of $\mathrm{Pd}$ calculated in an extended energy range by 
Müller and Wilkins [41]. It is shown that the fine structure of the BIS spectrum of $P d$ is strikingly similar to the $p$ partial density of states (DOS), but very different from the total DOS.

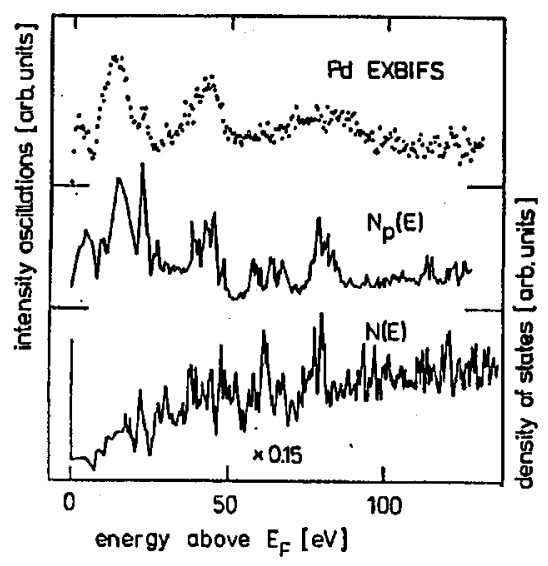

Fig. 5. Pd EXBIFS compared with the total and $p$ partial density of electron states in Pd.

\section{Single-scattering theory of EXBIFS}

Two theoretical approaches can be used for interpretation of X-ray bremsstrahlung isochromats: a "long-range order" theory based on the electron band structure calculations (e.g. [33, 42, 43]) and a "short-range order" theory considering scattering of the final-state electrons from the near neighbors of the central atom $[36,44]$. The both theories would yield the same results, if the first one included the lifetime of the electron state and the Debye-Waller factor, and if the second one took into account the multiple scattering.

Both theories are based on the muffin-tin potential approximation and therefore can separate the total bremsstrahlung cross-section into atomic-like and solid-state factors. The band structure approach is more suitable for the near-threshold BIS, where the Debye-Waller factor is negligible, than for the extended energy range. Below we shall outline the single-scattering theory and show that it is a very good method for interpretation of the extended structure of BIS.

The l-partial contribution to the atomic bremsstrahlung cross-section depends strongly on energy and atomic number. If one takes a simplified problem of hydrogen-like ions, then the radial matrix elements for continuum-bound transitions can be evaluated analytically. Within the limits of this simplification, we could easily calculate $n l$-subshell contribution to the ionic oscillator strength e.g. for $\mathrm{Ni}$ as a function of the energy (see Fig. 6) using the effective-charge approximation and the scaling property to the electron capture cross-sections calculated for hydrogen-like ions by Hahn and Rule [25]. The $n l$-partial cross-sections decrease 


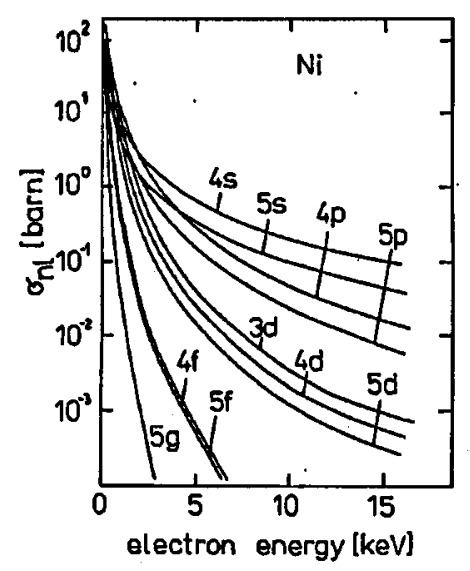

Fig. 6. The l-specific energy dependence of the cross-section for bremsstrahlung transition in $\mathrm{Ni}$ ion.

with increasing photon energy and the rate of decrease becomes larger with the $l$ number.

The relative partial probabilities of transitions approximated from the work of Hahn and Rule [25] were successfully used in calculations of bremsstrahlung near-edge spectra of $\mathrm{Ni}$ and $\mathrm{Mo}$ based on local partial densities of electron states weighted by the appropriate transition probabilities [27, 43].

Another example of band structure approach to BIS are theoretical calculations of the Ni and Pd near-edge BIS spectra [42]. In that work the transition-matrix elements were obtained using a computer program for X-ray photoemission valence band spectra [34].

First calculations of partial probabilities for bremsstrahlung transition in the extended energy range for neutral atoms were performed for $\mathrm{Cu}$ and $\mathrm{Pd}$ atoms by Šimůnek, Vackář and Sobczak [35]. Their results are presented in Table II. They established that the major contribution to the matrix element comes from a distance $\rho$ equal to about $0.01-0.1 \AA$, which is much smaller than the wavelength of isochromats. Therefore, the dipole approximation could be used for matrix element calculations. The fact that outgoing electron comes from the core region of the atom has crucial significance for the scattering theory of EXBIFS.

These results show that the ratios of the partial transition probabilities strongly depend on the atomic number and photon energy. The $s, p, d$ ratios for an element given and a fixed photon energy vary slowly within the final-state energy range $55-380 \mathrm{eV}$.

The results explain the fact that the high-energy BIS of $\mathrm{Cu}$ does not reflect the total DOS, but mostly the contributions from $s$ and $p$ partial density of states. The problem how to distinguish experimentally the both contributions seems to be unresolvable for $\mathrm{Cu}$ and $\mathrm{Ni}$, because both $s$ and $p$ partial densities of states in $\mathrm{Cu}$ and $\mathrm{Ni}$ have the main maxima positioned at nearly the same energies $[46,47]$. 


\section{TABLE II}

The relative ratios of the partial transition probabilities for BIS of $\mathrm{Cu}$ and $\mathrm{Pd}$ atoms [35] calculated for the self-consistent muffin-tin potentials of Moruzzi, Janak, and Williams [45]. The $f$-like probabilities are quite small and for this reason are not indicated.

\begin{tabular}{c|r|c|c|c|c|c|c}
\hline \hline$h \nu$ & $E_{f}$ & \multicolumn{3}{|c|}{$\mathrm{Cu}$} & \multicolumn{3}{|c}{$\mathrm{Pd}$} \\
\cline { 3 - 7 }$[\mathrm{eV}]$ & {$[\mathrm{eV}]$} & $s$ & $p$ & $d$ & $s$ & $p$ & $d$ \\
\hline 1487 & 54.4 & 1.00 & 3.47 & 1.83 & 1.00 & 4.48 & 6.95 \\
1487 & 163.3 & 1.00 & 3.40 & 1.76 & 1.00 & 4.41 & 6.38 \\
1487 & 272.2 & 1.00 & 3.30 & 1.76 & 1.00 & 4.34 & 6.02 \\
1487 & 380.9 & 1.00 & 3.20 & 1.77 & 1.00 & 4.27 & 5.77 \\
5415 & 54.4 & 1.00 & 1.46 & 0.19 & 1.00 & 2.75 & 1.34 \\
5415 & 163.3 & 1.00 & 1.46 & 0.20 & 1.00 & 2.77 & 1.31 \\
5415 & 272.2 & 1.00 & 1.46 & 0.22 & 1.00 & 2.74 & 1.31 \\
5415 & 380.9 & 1.00 & 1.45 & 0.24 & 1.00 & 2.72 & 1.31
\end{tabular}

The scattering theory defines the wave function of the final state not as a solution of the Schrödinger equation for the crystal, but as a modification of the atomic-like wave function by waves scattered by neighboring atoms.

Below we present the formula for EXBIFS based on the single-scattering and plane-wave approximation. For simplification we consider polycrystalline solids only and assume the potential to be spherically symmetrical inside the atomic sphere of radius $r_{0}$ and constant outside the sphere (so-called muffin-tin approximation). Furthermore, the simplification is made that the multiple scatterings are negligible. We assume that the size of an atom is small in comparison to the interatomic distance (plane-wave approximation).

In bremsstrahlung transition the final electron state can be described by a sum of spherical waves, over all symmetries $l$, originating at the atomic centre and characterized by phase shifts $\delta_{l}$ due to the potential at the central atom. In a solid, the outgoing spherical waves propagate out of the central atom toward the neighboring atoms. On the way, each of waves would have acquired a phase shift $\left(k r_{j}+\delta_{l}\right)$, where $r_{j}$ is the distance to the neighboring atom $j$. If we assume that $r_{j}$ is sufficiently larger than $r_{0}$, we can approximate the outgoing spherical wave by a plane wave in the vicinity of the neighboring atom $j$. This is the so-called small-atom or plane-wave approximation.

The scattering of this plane wave by the neighboring atom $j$ produces a spherical wave originating from the atom $j$ with the backscattering amplitude $f_{j}(k)$ being a complex number

$$
f_{j}(k)=\left|f_{j}(k)\right| \exp \left[\mathrm{i} \theta_{j}(k)\right],
$$

where $\theta_{j}(k)$ is the backscattering phase function of the $j$-th neighboring atom.

The scattered wave coming to the central atom can be approximated by a 
plane wave with a phase shift $k r_{j}$ and expanded in terms of spherical wave at the central atom with a phase shift $\delta_{l}$. The total phase shift of the l-partial wave returning to the central atom is $\left(2 k r_{j}+2 \delta_{l}+\theta_{j}\right)$.

For the purpose of calculating the bremsstrahlung rate we need only to consider the final state near the origin. The interference between the scattered and primary waves results in oscillation of square matrix elements $\left|M_{l}^{\text {solid }}\right|^{2}$ in the $k$ space

$$
\left|M_{l}^{\text {solid }}\right|^{2}=\left|M_{l}^{\text {atom }}\right|^{2}\left(1+\chi_{l}\right)
$$

where $\chi_{l}$ denotes the oscillating term

$$
\chi_{l}(k)=(-1)^{l} \sum_{j} \frac{N_{j}}{k r_{j}^{2}}\left|f_{j}(k)\right| \sin \left(2 k r_{j}+2 \delta_{l}+\theta_{j}\right) .
$$

The factor $(-1)^{l}$ can be put into the phase term, which is shown below in Eq. (8). The oscillating term $\chi_{l}$ is a discrete approximation of the following integral:

$$
\chi_{l}(k)=(-1)^{l} \frac{4 \pi}{k} \int_{0}^{\infty}|f(k, r)| \rho(r) \sin \left[2 k r+2 \delta_{l}+\theta(r)\right] \mathrm{d} r,
$$

where $4 \pi r^{2} \rho(r)$ is the radial distribution function. The Fourier transform enables extraction of the structure radial function $\rho(r)$, if the phase shifts and amplitude, and $\chi_{l}(k)$ are known.

The atomic vibrations decrease the amplitudes of oscillations by the factor $\exp \left(-2 \sigma_{j}^{2} k^{2}\right)$, if we assume harmonic vibrations only, where $\sigma_{j}^{2}$ is Debye-Waller's factor and $\sigma_{j}$ is the root-mean-square relative displacement. It should be noted that Debye-Waller's factor differs from the Debye-Waller factor for X-ray diffraction, because the coherent movement of atoms does not contribute to the Debye-Waller factor in the case of EXBIFS or EXAFS.

It is also necessary to introduce to the EXBIFS formula the damping factor $\exp \left(-2 r_{j} / \lambda\right)$ associated with the lifetime of the electron final state. In the case of EXAFS both the core-hole lifetime and photoelectron lifetime contribute to $\lambda$. This is not the case in EXBIFS, where there is no core hole. Therefore, in EXBIFS the $\lambda$ is simply the mean free path (MFP) of an electron for inelastic scattering.

The oscillating part $\chi$ of the total bremsstrahlung cross-section for a polycrystalline solid can be calculated as follows:

$$
\chi=\sum_{l} P_{l}^{\mathrm{at}} \chi_{l}
$$

where $P_{l}^{\text {at }}$ is the probability of bremsstrahlung transition to the final state with the symmetry $l$ in an atom.

Finally, we have got the basic formula for EXBIFS in the frame of the single-scattering theory

$$
\begin{aligned}
\chi(k) & =\sum_{j} \frac{N_{j}}{k r_{j}}\left|f_{j}(k)\right| \mathrm{e}^{-2 \sigma_{j}^{2} k^{2}} \mathrm{e}^{-2 r_{j} / \lambda} \\
& \times \sum_{l} P_{l}^{\mathrm{at}} \sin \left\{2 k r_{j}+2 \delta_{l}+\theta_{j}+\left[1-(-1)^{\prime}\right] \pi / 2\right\},
\end{aligned}
$$

where the normalized EXBIFS function is $\chi(k) \equiv\left(I-I_{0}\right) / I_{0}, I$ is the observed BIS intensity for the material, $I_{0}$ is a smooth BIS contribution from an isolated atom. 
The important quantities here are the l-partial probabilities of bremsstrahlung transitions in an atom.

Equation (8) can be used for calculation of EXAFS at the $K$ and $L_{1}$ absorption edges, if we put $l=1$ and $P_{l}^{\text {at }}=1$. One should take into account that the phase shift $\delta_{1}$ is slightly different for EXAFS than $\delta_{1}$ for EXBIFS due to a core hole at the central atom. In EXAFS the damping parameter $1 / \lambda=1 / \lambda_{\mathrm{h}}+1 / \lambda_{\mathrm{ph}}$ is associated with the core-hole lifetime and inelastic scattering of final state electrons. Here $\lambda_{\mathrm{h}}=v \tau=(2 E / m)^{1 / 2} \hbar / \Gamma_{\mathrm{h}}$ is the path of the photoelectron with the kinetic energy $E$ and the velocity $v$, which is passed during the core-hole lifetime $\tau$ related to the natural width $\Gamma_{\mathrm{h}}$ of the core level, and $\lambda_{\mathrm{ph}}$ is the mean free path of an electron for inelastic scattering.

We calculated the $\mathrm{Cu}$ and Pd EXBIFS by applying the single-scattering formula (8) and using values of transition probabilities for $\mathrm{Cu}$ and $\mathrm{Pd}$ from Table II. Only transitions to $s, p$, and $d$ final states were taken into account, because other transitions were negligibly weak. We calculated also $K$ EXAFS for $\mathrm{Cu}$ and $\mathrm{Pd}$. The numbers of atomic neighbors $N_{j}$ and distances $r_{j}$ were taken from crystallographic data. The backscattering amplitudes $\left|f_{j}\right|$ and backscattering phase shifts $\theta_{j}$ were taken the same for EXBIFS and EXAFS from the paper by Teo and Lee [48]. The central atom phase shifts $\delta_{l}$ were taken from the same paper, however they were chosen different for EXBIFS than for EXAFS. For the Cu and Pd EXBIFS we took the phase shifts of the Ni and Rh EXAFS, respectively. In practice, any phase function can be modified by $\pm 2 n \pi$, where $n=0,1,2,3 \ldots$ With this remark, we chose the domain of phase function values arbitrarily for the additive term $\pm 2 n \pi$. The phase shifts $2 \delta_{l}+\theta+\left[1-(-1)^{l}\right] \pi / 2$ at $l=0,1,2$ for the Cu and Pd EXBIFS, calculated using the phase data mentioned above, are plotted in Fig. 7. The result presented in Fig. 7 is very important, because it leads us to a theoretical conclusion
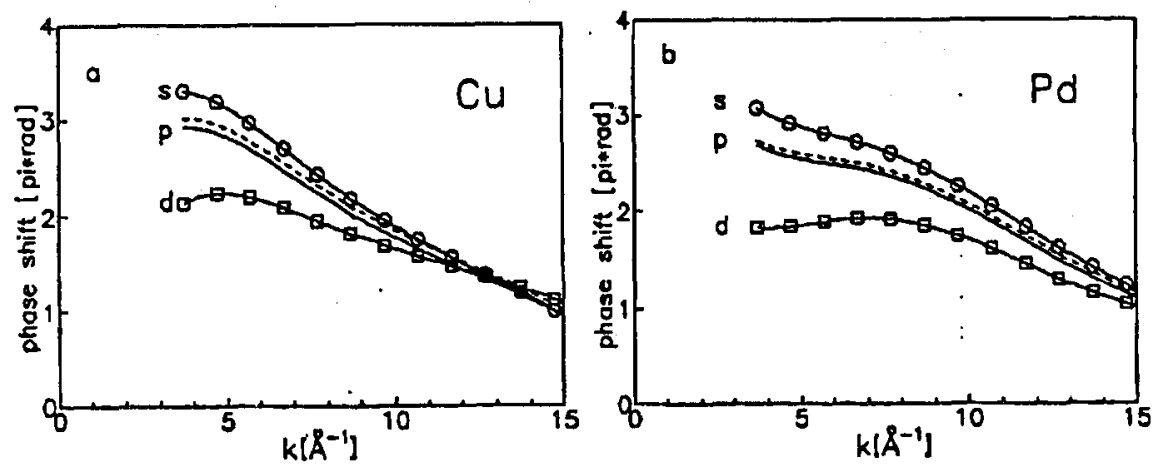

Fig. 7. EXBIFS phase shifts $2 \delta_{l}+\theta+\left[1-(-1)^{l}\right] \pi / 2$ at a big momentum $k$ (solid line) are nearly identical for $\mathrm{Cu}(\mathrm{a})$ and close to each other in the case of $\mathrm{Pd}(\mathrm{b})$.

that $s, p$, and $d$ oscillations contributed to EXBIFS should have the same phases at the large $k$ values. Therefore, the $l$-dependent probability of BIS transition should not be crucial for EXBIFS if $k$ is bigger than $10 \AA^{-1}$.

In the calculations the universal curve of mean free path of electrons [49] 
was approximated by the formula

$$
\lg _{10} \lambda=-0.067 x^{3}+0.911 x^{2}-2.84 x+3.31
$$

where $x=\lg _{10} E$, the mean free path $\lambda$ is in $\AA$ and the energy $E$ is in $\mathrm{eV}$.

It is very surprising that the Cu EXBIFS calculated by single-scattering approximation and shown in Fig. 8a is nearly identical to the Cu $K$ EXAFS shown in the same figure. Quite good similarity is also obtained for Pd EXBIFS and
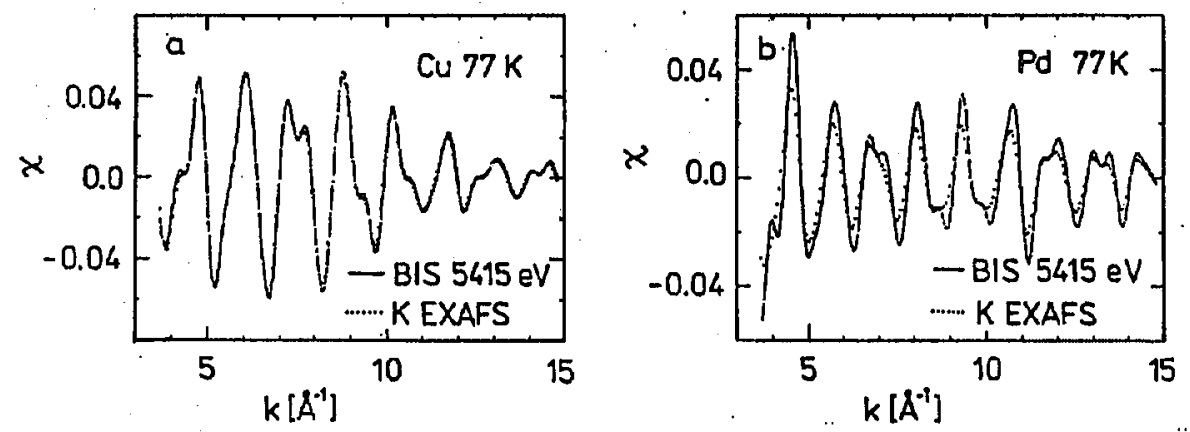

Fig. 8. EXBIFS at $5415 \mathrm{eV}$ (solid lines) and $K$ EXAFS (dots) calculated by single-scattering method for 5 shells at temperature $77 \mathrm{~K}$ : (a) for $\mathrm{Cu}$, (b) for $\mathrm{Pd}$.

Pd $K$ EXAFS (Fig. 8b). They lead us to the nontrivial conclusion that EXBIFS oscillations should not differ visually from $K$ EXAFS, if taken at the same temperature. In spite of quite big $s$ and $d$ contributions into EXBIFS, this striking similarity should occur for the $3 d$ and $4 d$ transition metals at the energy $5415 \mathrm{eV}$, and for the $3 d$ transition metals at the energy $1487 \mathrm{eV}$. For easier understanding of this phenomenon we have presented in Figs. 9 and 10 the first-shell $s, p$, and $d$ contributions to the $\mathrm{Cu}$ and Pd EXBIFS calculated for two different photon energies: 1487 and $5415 \mathrm{eV}$. In separate plots the $p$-type oscillation (solid line) has been compared with each oscillation of other type (dotted line) and the sum of oscillations (dotted line) as well.

It is seen in Fig. 9 that in the case of $\mathrm{Cu}$ the sum of oscillations is quite the same as the $p$-type oscillation. At the photon energy $5415 \mathrm{eV}$ the $d$-type oscillation is negligibly small in comparison to the $s$ and $p$ ones, and the quite big oscillation of $s$-type is not much different in phase relative to the $p$-type oscillation. At the photon energy $1487 \mathrm{eV}$ the $s$ - and $d$-type oscillations have opposite phases for low $k$ values and therefore their common contribution into the sum is small in comparison to the $p$ oscillation. For a big momentum $k$ the phases of all the $s$, $p$, and $d$ oscillations are not much different and therefore the sum of oscillations must agree well with the $p$ oscillation, independently on the photon energy.

The same good result was obtained for $\mathrm{Pd}$ and the photon energy $5415 \mathrm{eV}$, in spite of quite big $s$ and $d$ oscillations. The $s$ and $d$ oscillations have opposite phases and give together a negligible contribution to the sum at low $k$. We did not obtain such satisfactory agreement for $\mathrm{Pd}$ at the photon energy $1487 \mathrm{eV}$. The sum agrees better with the $d$ oscillation than with the $p$ one. 


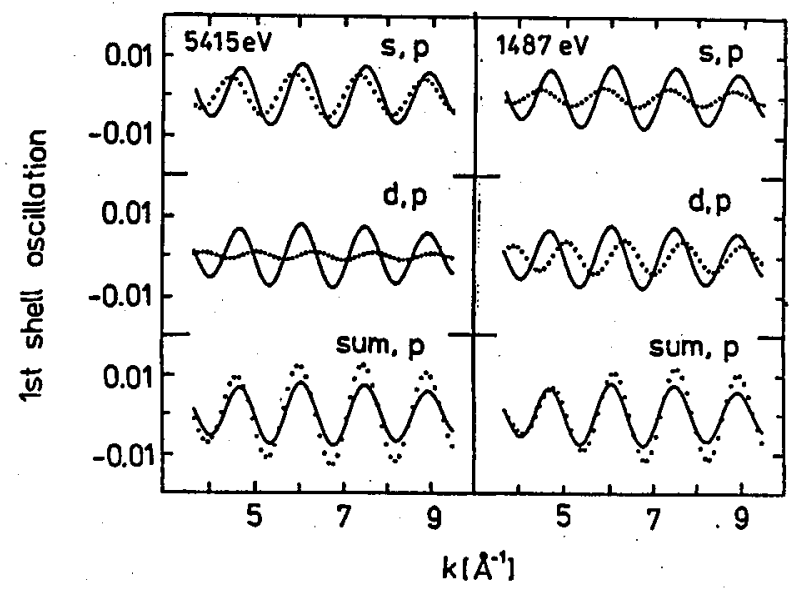

Fig. 9. Comparison between the p-type contribution (solid line), the $s$ - or $d$-type contributions (dotted line) and the sum (dotted line) calculated for the first-shell EXBIFS oscillations of $\mathrm{Cu}$ for two photon energies 5415 and $1487 \mathrm{eV}$.

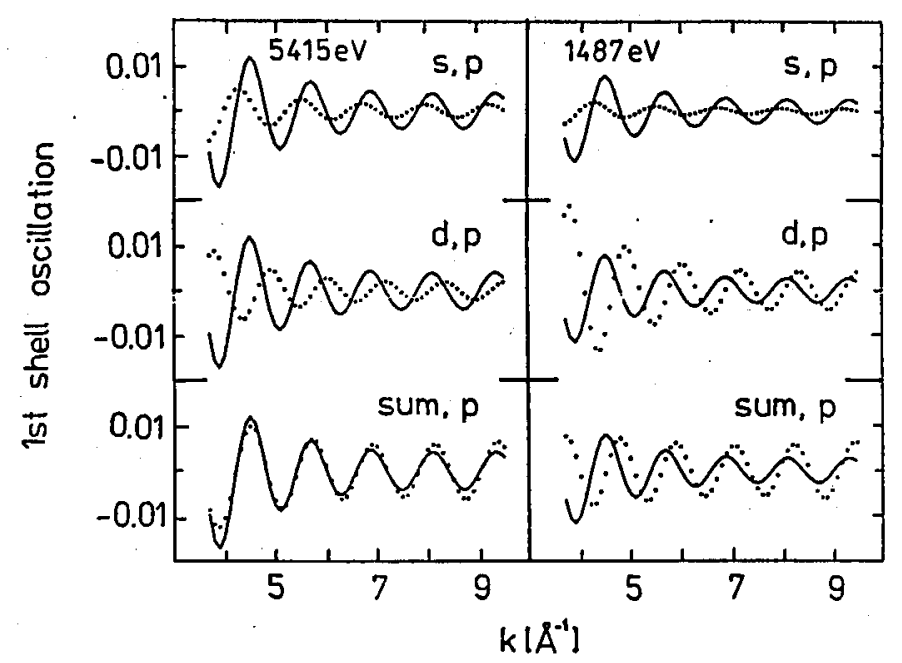

Fig. 10. Comparison between the p-type contribution (solid line), the $s$ - or $d$-type contributions (dotted line) and the sum (dotted line) calculated for the first-shell EXBIFS oscillations of Pd for two photon energies 5415 and $1487 \mathrm{eV}$.

We can easily estimate the temperature effect in EXBIFS using the single-scattering theory (Eq. (8)). Such studies cannot be done using a band-structure approach to EXBIFS. The theoretical values of the Debye-Waller factors $\sigma$ as a function of temperature are given for $\mathrm{Cu}$ and other metals in the work by Desjonquères and Tréglia [50]. As an example of temperature effect, results of 


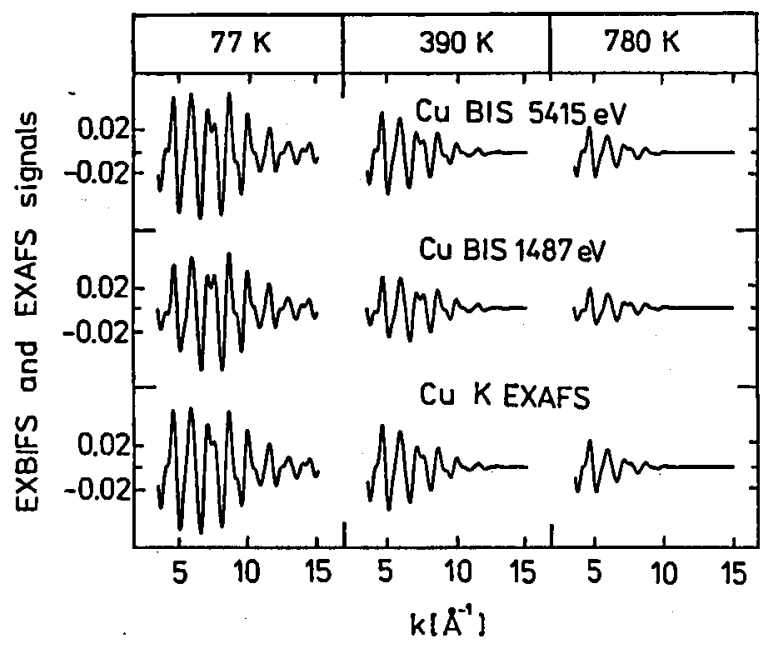

Fig. 11. Cu EXBIFS at photon energies 5415 and $1487 \mathrm{eV}$, and $\mathrm{Cu} K$ EXAFS calculated for three various temperatures.

EXBIFS and EXAFS calculations at three various temperatures are shown for $\mathrm{Cu}$ in Fig. 11. For our calculations we chose three values of the Debye-Waller factor $\sigma: 0.05,0.1$, and $0.14 \AA$. Such values correspond to the set of temperatures: $77,390,780 \mathrm{~K}$ for $\mathrm{Cu}$. It has been shown here using the single-scattering theory that temperature effects in EXBIFS and EXAFS are extremely strong for big $k$ values.

\section{Evaluation of interatomic distances from EXBIFS}

In the case when an EXBIFS spectrum is well approximated by one $\chi_{l}$ contribution, the oscillating term from Eq. (8) is equal to the following integral:

$$
\chi(k)=(4 \pi / k) \int_{0}^{\infty}|A(k, r)| \rho(r) \sin \left(2 k r+\Delta_{l}\right) \mathrm{d} r,
$$

where $\Delta_{l}$ is the $k$-dependent phase shift for the $l$-electron wave, $|A(k, r)|$ is the amplitude function, $4 \pi r^{2} \rho(r)$ is the radial distribution function and $\rho(r)$ is the radial structure function.

The Fourier transform of $\chi$ gives as a result a modified radial structure function $|g(r)|$, which differs from $\rho(r)$ because of the phase shift $\Delta_{l}$ and amplitude function $|A|$. The Fourier transform is calculated in the limited range from $k_{\min }$ to $k_{\max }$ using the following formula:

$$
g(r)=(2 / \pi)^{1 / 2} \int_{k_{\min }}^{k_{\max }} w(k) k^{n} \chi(k) \mathrm{e}^{\mathrm{i} 2 k r} \mathrm{~d} k,
$$

where $w(k)$ is a window function and $k^{n}$ is a weighting function.

It is well known that the limited range of integration causes the broadening of the modified radial structure function and produces numerous ripples around 
every peak of the Fourier transform. The ripples can be reduced using a window function smoothly setting the data to zero at $k_{\min }$ and $k_{\max }$. The lower limit $k_{\min }$ of the Fourier integral is usually chosen at a value of about $2 \AA^{-1}$ due to a strong nonlinearity of phase shifts for low $k$ values. The higher limit $k_{\max }$ is determined by experiment or chosen at a value where oscillations vanish due to Debye-Waller's factor. The narrower momentum range $\left(k_{\min }, k_{\max }\right)$ for the Fourier transform, the bigger broadening of the modified radial structure function $g(r)$.

If the $j$-th atomic shell is well separated in the range from $r_{\min }$ to $r_{\max }$ of the Fourier transform, the Fourier backtransform [51] in this range is used for evaluation of the amplitude $A_{j}(k)$ and phase $\varphi_{j}(k)$ of the sine-like oscillation $\chi_{j}(k)$ :

$$
\chi_{j}(k)=A_{j}(k) \sin \left[\varphi_{j}(k)\right] \text {. }
$$

(The Fourier backtransform can be also applied for the Fourier filtering in any $r$ range.) From the phase $\varphi_{j}(k)$ being a total phase for the $j$-th shell

$$
\varphi_{j}(k)=2 k r_{j}+\Delta_{l}(k)
$$

one can derive the radius $r_{j}$, if the phase shift $\Delta_{l}$ is known.

The last formula was used for determination of the interatomic distance $r_{1}$ from EXBIFS of several fcc metals. The Fourier transform moduli $|\mathrm{FT}|$ of the EXBIFS and $K$ EXAFS spectra are shown in Figs. 12 and 13. The first-shell peak at $d_{\mathrm{FT}}$ is well resolved in the Fourier transform, however it is shifted down to a lower value by $0.2-0.4 \AA$ in comparison to the crystallographic distances $d_{\text {cryst }}$, which are shown in Table III. In general, it changes a little according to $E_{0}, k_{\min }$ and $k_{\max }$ values. A good qualitative agreement is observed in the feature of FT between EXBIFS and EXAFS of $\mathrm{Co}, \mathrm{Ni}, \mathrm{Cu}$, and $\mathrm{Pd}$, if they are transformed in the same $k$ range.

TABLE III

Nearest-neighbors distances for several fcc metals obtained from EXBIFS using a Fourier transform $\left(d_{\mathrm{FT}}\right)$ and using a Fourier backtransform and applying EXAFS phase shifts $\left(d_{\mathrm{ps}}\right)$, compared to crystallographic distance $d_{\text {cryst }}$ (values in $\AA$ ).

\begin{tabular}{c|c|c|c}
\hline \hline & $d_{\mathrm{FT}}$ & $d_{\mathrm{ps}}$ & $d_{\text {cryst }}$ \\
\hline $\mathrm{Ni}$ & 2.20 & 2.49 & 2.49 \\
$\mathrm{Cu}$ & 2.25 & 2.47 & 2.55 \\
$\mathrm{Co}$ & 2.10 & 2.47 & 2.51 \\
$\mathrm{Pd}$ & 2.50 & 2.73 & 2.75
\end{tabular}

Using the Fourier backtransform of the first-shell peak, the phase $\varphi_{1}(k)$ was evaluated for EXBIFS and EXAFS of metals studied. Assuming the EXAFS data as the "model system" with the interatomic distance $d_{\text {cryst }}$ and the EXBIFS data as the "unknown system" with the interatomic distance $d_{\mathrm{ps}}$, we have obtained from formula (13) the nearest-neighbors distance $r_{1}$ applying EXBIFS's phase $\varphi_{1}(k)$ and 




Fig. 12. Moduli of Fourier transforms of EXBIFS and $K$ EXAFS for $\mathrm{Cu}, \mathrm{Ni}$, and Co $[52]$.

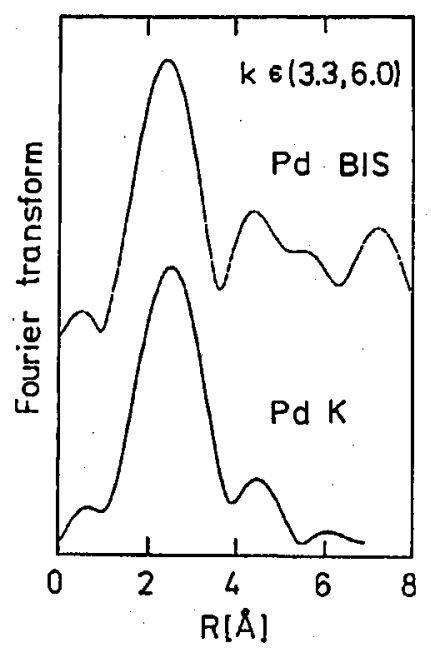

Fig. 13. Moduli of Fourier transforms of Pd EXBIFS (upper curve) and Pd $K$ EXAFS (lower curve) calculated in the same $k$ range [53]. 
EXAFS's phase shift $\Delta_{l}(k)$. The position $E_{0}$ of BIS relative to EXAFS had been shifted down by $\Delta E=5,1,5,4 \mathrm{eV}$ for $\mathrm{Co}, \mathrm{Ni}, \mathrm{Cu}$, and $\mathrm{Pd}$, respectively, to get the most constant value $r_{1}$ as function of $k$. The interatomic distances $d_{\mathrm{ps}}$ obtained from EXBIFS using EXAFS's phase shifts and shown in Table III are smaller than the crystallographic distance $d_{\text {cryst }}$ by values of $0.04,0.08$, and $0.02 \AA$ for $\mathrm{Co}, \mathrm{Cu}$, and $\mathrm{Pd}$, respectively, and unchanged for $\mathrm{Ni}$. It is not clear at the moment why the EXBIFS phase of $\mathrm{Ni}$ is nearly identical to that of $K$ EXAFS.

\section{Summary}

Original concept of investigations of the extended X-ray bremsstrahlung isochromat fine structure has been presented in details both theoretically and experimentally. The BIS measurements at the photon energy $5415 \mathrm{eV}$ were performed up to about $250 \mathrm{eV}$ above the bremsstrahlung threshold for several metals with the fcc structure like $\mathrm{Cu}, \mathrm{Ni}, \mathrm{Co}, \mathrm{Ag}$, and $\mathrm{Pd}$ using a home-made X-ray spectrometer. Intensity oscillations in BIS spectra were fairly well observed for all samples studied, and found to be similar to $K$ EXAFS.

A theoretical model of EXBIFS based on the single-scattering theory has been formulated here. A clear picture of EXBIFS is given here as follows:

a) EXBIFS structure is related neither to the total density of electron states, nor characteristic energy loss spectrum. It has been established in this work that the EXBIFS structure is similar to the $p$-type partial density of states.

b) X-ray bremsstrahlung transition is localized in the vicinity of the nucleus and therefore the condition of the point origin of electron wave necessary in the single-scattering treatment is fulfilled.

c) Probabilities of bremsstrahlung transitions to final states with various symmetry $s, p, d, f$ depend upon the photon energy and atomic number of material studied, and are biggest for $p$-type final states and negligibly small for $f$-type ones in the case of photon energy $5415 \mathrm{eV}$, and $\mathrm{Cu}$ and $\mathrm{Pd}$ atoms.

d) The single-scattering calculations for first-shell oscillations of EXBIFS show that the sum of $s, p, d$ contributions becomes $p$-like, because $s$ and $d$ contributions are in opposite phases and compensate each other. Therefore the theoretical EXBIFS is obtained nearly identical to the theoretical $K$ EXAFS.

e) The single-scattering model of EXBIFS has been successfully applied in explanation of temperature effects resulting in smearing of oscillations for bigger $k$ values.

The most important conlusion of this work is that EXBIFS has been established as a new method of evaluation of the interatomic distances, at least for one component material, using the single-scattering theory and the Fourier transform. Results of our work have shown that the Fourier transform without corrections for phase shifts makes it possible to evaluate the interatomic distances very roughly with a systematic error of about $20 \%$. This accuracy is improved up to some $3 \%$, if EXAFS's phase shifts are used for correction. We hope that in the near future the accuracy should be enhanced to $1 \%$ or better owing to development of BIS experiment and theory. 
It is interesting to note that damping of EXBIFS amplitudes can inform us about either the temperature or structural disordering in material studied.

\section{Acknowledgments}

This article is dedicated to Professor J. Auleytner, my teacher, on the occasion of his 70th birthday.

I would like to thank Professor S. Mobilio for EXAFS spectra and EXAFS computer programs, Mrs. Z. Bihatowicz for the technical assistance.

\section{References}

[1] W.C. Röntgen, Sitz.-Ber. der Würzburger Akad., p. 137 (1895) (reprinted in Ann. d. Phys. 64, 1 (1898)).

[2] A. Einstein, Ann. d. Phys. 17, 145 (1905).

[3] W. Duane, F.L. Hunt, Phys. Rev. 6, 166 (1915).

[4] P. Ohlin, Ph.D. dissertation, Uppsala Universitets Arsskrift 1 (1941); Ark. Mat. Astr. Fys. Ser. A 29, 1 (1942); Nature 152, 329 (1943).

[5] J.A. Bearden, G. Schwarz, Phys. Rev. 59, 934 (1941).

[6] J. Lindhard, Ark. Mat. Astr. Fys. Ser. B 31, 1 (1945).

[7] B.R.A. Nijboer, Physica 12, 461 (1946).

[8] W. Wien, Gött. Nachr., p. 598 (1907); Ann. d. Phys. 23, 434 (1907).

[9] W. Friedrich, P. Knipping, M. Laue, Münchener Ber., p. 303 (1912); Ann. d. Phys. 41, 971 (1913).

[10] W.L. Bragg, Proc. Cambr. Phil. Soc. 17, 43 (1912); Nature 90, 410 (1912).

[11] C.T. Urley, Phys. Rev. 11, 401 (1918).

[12] H. Kuhlenkampff; Dissertation, München 1922; Ann. d. Phys. 69, 548 (1922).

[13] H.A. Kramers, Philos. Mag. 46, 836 (1923).

[14] A. Sommerfeld, Ann. d. Phys. 11, 257 (1931).

[15] A. Nilsson, Ark. Fys. 6, 513 (1953).

[16] C. Gerthsen, L. Albert, Z. Angew. Phys. 6, 196 (1954).

[17] J. Kessler, K. Ulmer, Z. Phys. 159, 443 (1960).

[18] J. Auleytner, B. Lidén, Ark. Fys. 23, 41 (1962).

[19] J. Eggs, K. Ulmer, Z. Angew. Phys. 20, 118 (1965).

[20] G. Böhm, K. Ulmer, Z. Phys. 228, 473 (1969).

[21] H. Merz, Phys. Status Solidi A 1, 707 (1970).

[22] G. Mechelke, K. Ulmer, Phys. Status Solidi A 19, 573 (1973).

[23] R.J. Liefeld, A.F. Burr, M.B. Chamberlain, Phys. Rev. A 9, 316 (1974).

[24] V. Dose, Appl. Phys. 14, 117 (1977).

[25] Y. Hahn, D.W. Rule, J. Phys. B, At. Mol. Phys. 10, 2689 (1977).

[26] J.K. Lang, Y. Baer, P.A. Cox, Phys. Rev. Lett. 42, 74 (1979).

[27] C.C. Chu, P.E. Best, Phys. Rev. 19, 3414 (1979). 
[28] F. Riehle, Phys. Status Solidi B 98, 245 (1980).

[29] E. Sobczak, R. Goldberg, J. Pelka, J. Auleytner, in: Inner-Shell and X-Ray Physics of Atoms and Solids, Eds. D.J. Fabian, H. Kleinpoppen, L.M. Watson, Plenum, New York 1981, p. 529.

[30] J.B. Pendry, J. Phys. C, Solid State Phys. 14, 1381 (1981).

[31] J. Unguris, A. Seiler, R.J. Celotta, D.T. Pierce, P.D. Johnson, N.V. Smith, Phys. Rev. Lett. 49, 1047 (1982).

[32] W. Speier, J.C. Fuggle, R. Zeller, M. Campagna, in: EXAFS and Near Edge Structure III, Eds. K.O. Hodgson, B. Hedman, J.E. Penner-Hahn, Springer, Berlin 1984, p. 496; W. Speier, T.M. Hayes, J.W. Allen, J.B. Boyce, J.C. Fuggle, M. Campagna, Phys. Rev. Lett. 55, 1693 (1985).

[33] E. Sobczak, J. Auleytner, Phys. Rev. B 37, 6251 (1988).

[34] W. Speier, J.C. Fuggle, P. Durham, R. Zeller, R.J. Blake, P. Sterne, J. Phys. C, Solid State Phys. 21, 2621 (1988).

[35] A. Šimůnek, J. Vackáŕ, E. Sobczak, Phys. Rev. B 38, 8515 (1988).

[36] A. Simůnek, O. Sipr, J. Vackáŕ, Phys. Rev. Lett. 63, 2076 (1989).

[37] B. Reihl, J.H. Coombs, J.K. Gimzewski, Surf. Sci. 211/212, 156 (1989).

[38] K. Tsutsumi, H. Nakamori, in: X-Ray Spectra and Electronic Structure of Matter, Vol. 1, Eds. A. Faessler, G. Wiech, Fotodruck Frank Ohg, Munich 1973, p. 100.

[39] I. Davoli, S. Stizza, A. Bianconi, M. Benfato, C. Furlani, V. Sessa, Solid State Commun. 48, 475 (1983).

[40] E. Sobczak, J. Auleytner, J. Phys. (France) 48, coll. C9, suppl. 12, 1121 (1987).

[41] J.E. Müller, J.W. Wilkins, Phys. Rev. B 29, 4331 (1984).

[42] W. Speier, J. Phys. C, Solid State Phys. 21, L1183 (1988).

[43] M.T. Czyżyk, K. Ławniczak-Jabłońska, S. Mobilio, Phys. Rev. B 45, 1581 (1992).

[44] O. Sipr, J. Vackár̆, A. Simůnek, Phys. Rev. B 44, 4832 (1991).

[45] V.L. Moruzzi, J.F. Janak, A.R. Williams, Calculated Electronic Properties of Metals, Pergamon, New York 1978.

[46] R.C. Albers, A.K. McMahan, J.E. Müller, Phys. Rev. B 31, 3435 (1985).

[47] F. Szmulowicz, D.M. Pease, Phys. Rev. B 17, 3341 (1978).

[48] B.K. Teo, P.A. Lee, J. Am. Chem. Soc. 101, 2815 (1979).

[49] I. Lindau, W.E. Spicer, J. Electron Spectrosc. Relat. Phenom. 3, 409 (1974).

[50] M.-C. Desjonquères, G. Tréglia, Phys. Rev. 34, 6662 (1986).

[51] P.A. Lee, P.H. Citrin, P. Eisenberger, B.M. Kincaid, Rev. Mod. Phys. 53, 769 (1981).

[52] E. Sobczak, J. Auleytner, S. Mobilio, A. Balerna, O. Smotlacha, Physica B 158, 553 (1989).

[53] E. Sobczak, S. Mobilio, in: Proc. 2nd Europ. Conf. on Progress in X-Ray Synchrotron Radiation Research, Roma 1989, Eds. A. Balerna, E. Bernieri, S. Mobilio, Italian Physical Society, Bologna 1989, p. 149. 